\title{
Experimental Study of the Dispersion Beneath Liquid Sprayers in the In- tersection Area of Jets on a Horizontal Plate
}

\author{
Kheireddine CHOUAL*, Redouane BENZEGUIR**, Mohamed TEBBAL*** \\ * Gas Fuels and Environment Laboratory, Mechanical Engineering Faculty, University of Sciences and the Technology - \\ Oran, USTO-MB, BP 1505, El-M'Naouer, Oran 31000, Algeria, E-mail: choual_kheireddine@yahoo.com \\ ** Gas Fuels and Environment Laboratory, Mechanical Engineering Faculty, University of Sciences and the Technology - \\ Oran, USTO-MB, BP 1505, El-M'Naouer, Oran 31000, Algeria, E-mail: benzeguir_redouane@yahoo.fr \\ ***Gas Fuels and Environment Laboratory, Mechanical Engineering Faculty, University of Sciences and the Technology \\ -Oran, USTO-MB, BP 1505, El-M'Naouer, Oran 31000, Algeria, E-mail: tebbal_mohamed@yahoo.fr
}

cross $^{\text {ref }}$ http://dx.doi.org/10.5755/j01.mech.23.6.17243

\section{Nomenclature}

$a$ - large diameter of the sprayer aperture, $\mathrm{mm} ; b$ - small diameter of the sprayer aperture, $\mathrm{mm}$; $D_{\text {large }}$ - diameter of the impacting zone, mm; $D_{\text {overlap }}$ - overlapping zone width, mm; $F$ - section area of the sprayer aperture, $\mathrm{mm}^{2} ; g$ - fluid dispersion, $\mathrm{mm}^{3} / \mathrm{mm}^{2} . \mathrm{s} ; \bar{g}$ - average dispersion, $\mathrm{mm}^{3} / \mathrm{mm}^{2} . \mathrm{s}$; $g_{\max }$ - maximum fluid dispersion, $\mathrm{mm}^{3} / \mathrm{mm}^{2} . \mathrm{s} ; g_{\text {exp }}$ - fluid dispersion obtained by experiments, $\mathrm{mm}^{3} / \mathrm{mm}^{2} . \mathrm{s} ; g_{\text {the }}$ - fluid dispersion obtained by summation law of both jets, $\mathrm{mm}^{3} / \mathrm{mm}^{2}$.s; $H$ - distance between sprayer and plane surface, $\mathrm{mm} ; H_{\text {overlap }}$ - overlapping area height, $\mathrm{mm} ; h$-depth of the sprayer slit, $\mathrm{mm} ; K$ - dimensionless number characterizes relation between dispersions; $K_{1}$ - hydrodynamic statistical parameter, $\mathrm{kg} / \mathrm{mm}^{2} . \mathrm{s} ; K_{2}$ - geometrical statistical parameter, $\mathrm{mm} ; L$ - Nozzles spacing, $\mathrm{mm}$; $\mathrm{n}$ - integer represents number of points; $R$ - inner radius of the sprayer channel, $\mathrm{mm} ; S$ section of the recovery tube, $\mathrm{mm}^{2} ; V$ - volume of recovered fluid, $\mathrm{mm}^{3} ; x_{\mathrm{i}}$ - coordinate on the $x$ axis; $y_{\mathrm{i}}$ - coordinate on the $y$ axis; $x_{\max }$ - radius of the impacting water along the $x$ axis, mm; $y_{\max }$ - radius of the impacting water along the $y$ axis, mm;

Greek Symbols -

$\mathrm{A} P$ - pressure variation, $\mathrm{MPa} ; \varphi$ - opening angle of the sprayer, deg; $\delta$ - thickness of the bottom's sprayer, mm; $\tau$ time, $\mathrm{s} ; \rho$ - mass density, $\mathrm{kg} / \mathrm{m}^{3} ; \sigma_{x}$ - variance; $\sigma_{y}$ - variance; $\xi$-dimensionless geometrical parameter.

\section{Introduction}

The transformation of water into a dispersion of small droplets is necessary for many industrial processes. Droplets are produced in nature and in a wide range of scientific and technological applications, it can be natural like a dew, rain, marine and ocean waves or artificial like droplets produced for the cooling of the highly heated plates (iron and steel industry), paint guns, inkjet printing applications, materials processing.

Spray is usually considered as a system of droplets submerged in a continuous gaseous phase in industrial cooling systems [1], it can be products in different manners. All forms of pressure nozzles evacuate fluid at high velocity in surrounding gas (usually air). Atomization and sprays are used in a wide range of industries: mechanical, science of materials, metallurgy, chemicals, aerospace, civil engineer- ing, forestry, environmental protection, agriculture, pharmaceutical, medical, food, weather, production of electricity, automotive and many other fields $[2,3]$, The main reason for disperse liquid into droplets is the gain resulting from the increase of the liquid surface area. This is the case for many processes, particularly for the cooling of plate highly heated in iron and steel industry, field of our research.

For this purpose, the cooling of plates highly heated beneath sprayers has known, in reliability of materials, an important development in recent decades. The desired materials (steels) with specific characteristics depends significantly on the cooling modes of the highly heated plates. Namely, the dispersion is a key factor in the spraying processes; it depends on a group of parameters including geometric parameters of spray-nozzles (the opening angle of the sprayer, thickness of the bottom's sprayer and inner radius of the sprayer channel) and hydrodynamic characteristics of jets. Often the dispersion is expressed as a flux rate per unit area, which is equivalent to a flux density $\left(\left(\mathrm{m}^{3} / \mathrm{s}\right) / \mathrm{m}^{2}\right)$. Furthermore, the sprayers are designed in multiple spray in industrial cooling systems to provide a uniform distribution of spray characteristics such as the volume flow or the spray impact. However, overlapping areas appear between fields of pulverisation, which affect the heat transfer and quality of the product obtained (steel or iron), in this sense, the analysis of the overlaps areas becomes important.

The importance of this study is from a constant need to evaluate experimentally the behaviour of the hydrodynamic parameters of two identical sprays in the intersection jets area on a horizontal plate. Seven pair of hydraulic atomizers were used with different hydrodynamic parameters; the aim is to analyse the behaviour of the water dispersion in the intersection area of jets and check the summation law of both jets for the atomizers selected.

Generally, the understanding of the fluid dispersion beneath sprayers remains a problem tackled and the knowledge in this area of research is not very large because of the complexity and the big number of parameters influencing the dispersion of the liquid that is why it is necessary to review some of the major works concerning the effects of hydrodynamics and geometrics parameters of jets on heat transfer to have a good knowledge of the fluid dispersion. 
The experimentation on the cooling jets requires the right choice of the hydrodynamic and geometric parameters such as; distance between sprayers and heated plate, nozzle spray angle, nozzles spacing, pressures rate and velocity of fluid [4], while, a number of authors have studied the effects of geometric and hydrodynamic parameters on the cooling of highly heated material.

Tebbal and Mzad lead a hydrodynamic study of a water jet dispersion beneath liquid sprayers, the authors determined an empirical result defining the dispersion profile of a water jet beneath liquid sprayers over a plane surface including the determining geometric and hydrodynamic parameters such as the nozzle's geometrical characteristics and pressure variation of liquid through the sprayer. The same authors lead another study in the range of ejected fluid pressure between 1 bar and 3 bars to evaluate the influence of the function $\mathrm{g}(\mathrm{x}, \mathrm{y})$, of dispersed water, on heat transfer variations across the surface at the temperature of 600C [6]. Cheng, et al. studied experimentally the effects of spray height, nozzle spray angle, inlet pressure and spray incident angle on heat transfer of spray cooling. The authors deduced that heat transfer performance can be optimized by a smaller spray angle nozzle, which sprays at a smaller spray height and a higher inlet pressure, while the effect of incident angle on heat transfer depends on nozzle spray angle and the definition of nozzle distance to surface. Visaria and Mudawar studied experimentally the effect of the inclination angles, flow rates, and subcoolings on a heated surface, the authors proposed correlation to accurately predict the effects of orientation angle on $\mathrm{CHF}$ for different nozzles and operating conditions. Chen, et al. studied the effects of the three hydrodynamic parameters (mean droplet size, droplet flux, and droplet velocity) on critical heat flux (CHF) while these parameters were systematically varied. The effect of each parameter was studied while keeping the other two nearly constant. The mean droplet velocity $(V)$ had the most dominant effect on CHF and the heat transfer coefficient, followed by the mean droplet flux $(N)$. The Sauter mean diameter $\left(d_{32}\right)$ did not appear to have an effect on CHF.Chen, et al (2002). , Celata, et al. studied the effect of drop velocity and surface inclination on a hot surface, authors experiment the behaviour of one drop impinging on a hot surface by varying the surface temperature, the drop velocity and the position of the surface (horizontal and a inclined $45^{\circ}$ ). It is concluded that to achieve the maximum possible $\mathrm{CHF}$ while using the minimum quantity of water, it is desirable to select nozzles that produce as small a droplet diameter with as high a velocity as possible and the inclination of the surface decreases the critical value of the temperature above which the surface is not rewetted.

The impact of geometric parameters of the sprayers was also broached by researchers cautiously, Abbasi, et al. postulated that the local normal pressure exerted by the spray onto the heated surface can be used to obtain the local heat transfer coefficient. Experiments were carried out using data obtained from hollow cone, full cone, and linear sprays at four nozzle pressures and three stand-off distances. A correlation between the pressure and heat transfer coefficient was determined from the data, then used to "predict" the heat transfer. Other experiments were performed to evaluate the spray cooling performance of three different hollow cone spray nozzles [13], for all tests, copper surface was utilized as the heater and tests were carried out at near atmospheric pressure conditions. All three nozzles were tested at various flow rates and nozzle-to-heater distances and the results were compared. It was been deduced that changing the nozzle-to-heater distance affects heat transfer rates more than critical heat flux (CHF). HORSKÝ and RAUDENSKÝ present an experimental method for measurement of heat transfer parameters of commonly used mist nozzles. The nozzles were tested for pressure setting, for influence of casting speed, for behaviour in the overlapping areas. The tests provide the description of heat transfer coefficient on the cooled steel surface.

Other studied were focused on the effects of hydrodynamic and geometric parameters of the sprayers on multijet cooling and on the behaviour of the fluid dispersion in the intersection area of jets. Hou, et al. studied the characteristics of multi-nozzle spray cooling using CFD method based on the fundamentals of air flow and liquid droplet collision dynamics, the simulations were performed using a Euleriane-Lagrangian approach. Focus was placed on revealing the flow behavior with multiple nozzles; the authors concluded that the nozzle inlet pressure and the mass flux influenced the Sauter Mean Diameter of droplets and the mass weighted average droplet velocity. Jung, et al. , Jurng, et al. investigated on the overlapping area of jets, the authors studied the behavior of the axial average velocity, the radial velocity and the average size of droplets according to probability density function (PDF) of the droplets size using imaging measurements. Zhou, et al. carried out an investigation of the influence of gas flow rate and liquid flow rate on the droplet sizes and velocities produced by a linear atomizer. The authors deduced that the droplet size decreases with an increase in atomizing gas pressure, the droplet velocity increases with atomizing gas pressure and the liquid height inside the crucible has little influence on liquid flow rate. Patrick, et al. effectuated measurements of pressures, flow rates and distributions in the two directions, transversal and longitudinal with both individual and multi nozzles, authors used two sprayers that produce flat jets and concluded that the dispersion in the intersection area of jets is not additive. However, Hall and Mudawar, Hattel and Pryds assumed that the volumetric spray flux contributed by two adjacent nozzles was additive at all $(\mathrm{x}, \mathrm{y})$ locations and the spatial distribution model of the volumetric spray flux was used to optimize nozzle spacing. Mzad and Elguerri lead an hydrodynamic investigation using a program of simulation based on the experimental correlations of pulverized water in the intersection area of jets. Authors used the principle of summation law of both jets and highlighted the influence of the hydrodynamic parameters by varying the water pressure AP between 0.2 and $0.9 \mathrm{MPa}$, which gives a very wide operating range. In addition, other parameters such as the nozzle's orifice-to-surface distance, $\mathrm{H}$, and the nozzle opening angle, $\mu$, are also highlighted by authors.

\section{Description of test bench and conditions of experiments}

The experiments on crossed jets were carried out on a test bench (Fig. 1) composed by a set of devices ; 1 valve. 2 - water container. 3 - container with fixed sprayers on the crossbar. 4 - crossbar fixation allowing movement of the sprayers 5 - fixation allowing the vertical and horizontal displacements of sprayers. 6 - battery pack of graduated glass tubes to quantify the fluid dispersion 
through the sprayers. 7 - manometers. $H$ - Height between sprayer and plate. $L$ - Distance between centers of sprayers.

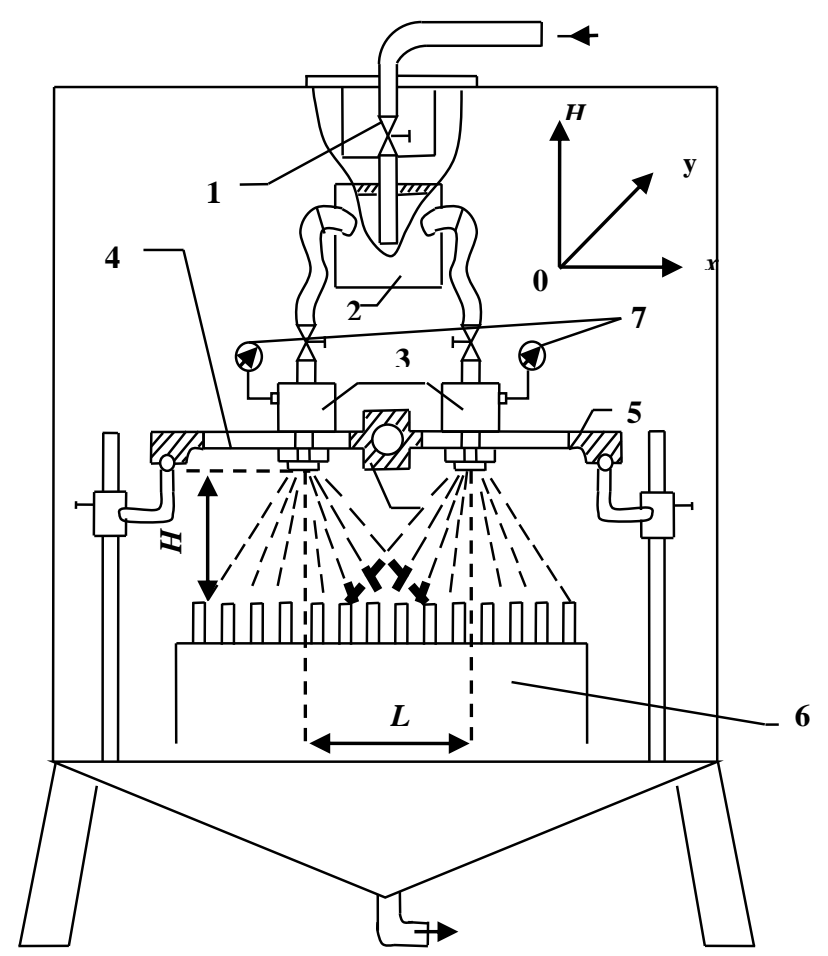

Fig. 1 Experimental test bench

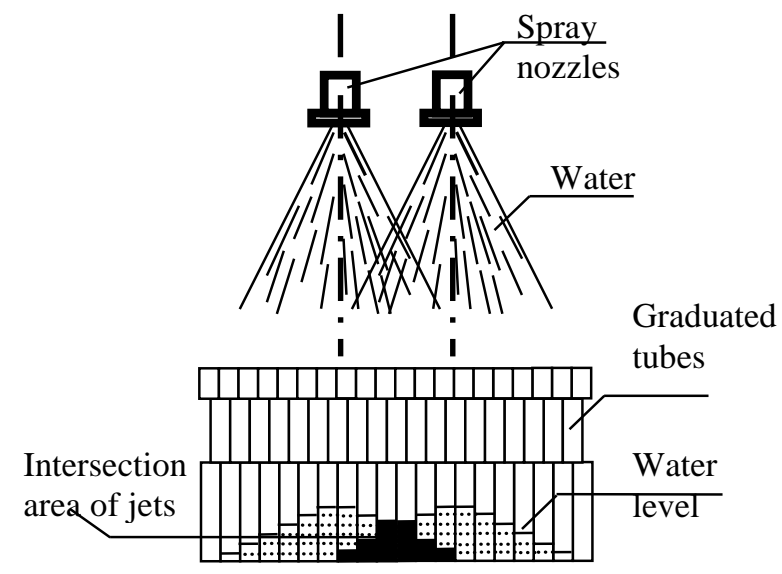

Fig. 2 Battery of graduated tubes for recovering the volume of the dispersed liquid

The graduated glass tubes allow to read the recovering volume $V$ of water dispersed per unit of time (Figs. 1 and 2) the fluid dispersion ( $g$ ) for each sprayer was calculated by the following relation:

$$
g=\frac{V}{S \tau},
$$

where $S$ represents the section of the graduated tube area and $\tau$ is time in seconds. Then the distance in the overlapping area of jets $D_{\text {overlap }}$ (Fig. 4 ) was given by de following relation:

$$
D_{\text {overlap }}=D_{l \text { arge }}-L,
$$

where $D_{\text {overlap }}$ is the diameter in the intersection jets area on a horizontal plate; $D_{\text {large }}$ is the diameter of the impacting water area by one sprayer and $L$ is the nozzles spacing (Fig. 4).

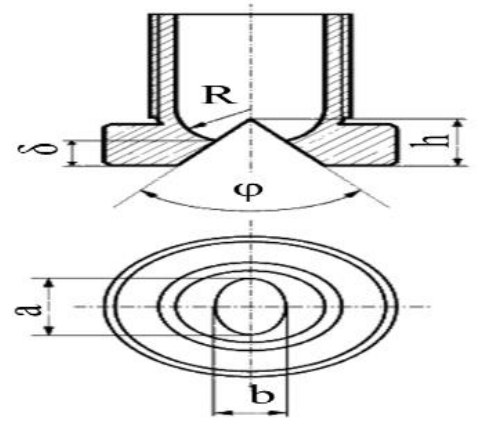

Fig. 3. Sprayer cut

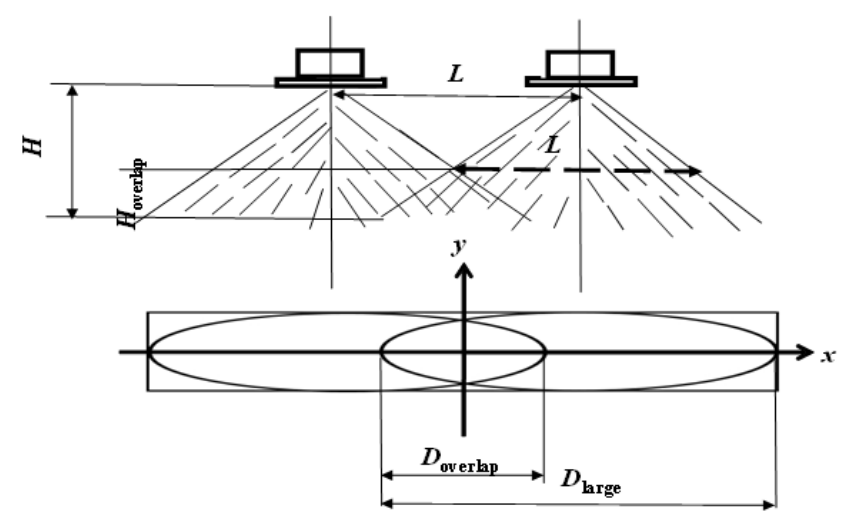

Fig. 4 Representation of intersecting jets

The hydrodynamics parameters ( $A P, L, H, H_{\text {overlap}}$, $D_{\text {large }}$ and $\left.D_{\text {overlap }}\right)$ were selected close to the actual values used in industrial cooling systems (Fig. 4). Where, the pressure of sprayers $A P$ varies from $0.05 \mathrm{MPa}$ to $0.3 \mathrm{MPa}$ with step of 0.05 , the distance between sprayer and plane surface $H$ varies between 385 and $450 \mathrm{~mm}, H_{\text {overlap }}$ depends on the conditions of the experiment, $D_{\text {large }}$ was given by the study of Tebbal and Mzad as following:

$$
D_{l \text { arge }}=48240 \cdot K_{2}^{-0.468}
$$

and

$$
K_{2}=H \cdot \operatorname{tg}(\varphi / 2)
$$

where $\varphi$ is the opening angle of the sprayer (Fig. 3 and TAB.01) and the nozzles spacing $L$ was calculated to maintain $D_{\text {overlap }}$ between 180 and $220 \mathrm{~mm}$ by the relation (2) as described above. (Fig. 4).

The sprayer used is in the form of a cylindrical channel with a spherical bottom, a conical slot is formed inside to govern the dispersion of liquid (Fig. 3).

Atomizers used in experiments produce elliptical dispersions of water droplets on horizontal plate (Fig. 4). The liquid dispersion depends to the geometrical parameters of the spray nozzles, $(\varphi, h, b, a, F$ and $\xi$ ) (Table 1$)$, as well as the hydrodynamic conditions such as: the height between the sprayer and the cooled surface $(H)$, the variation of the spraying pressure $(A P)$, the nozzles spacing $(L)$ (Fig. 4), and perhaps other parameters such as the sprayers angle compared with the surface of plate. 
Geometrics parameters of the sprayers used in experiment

\begin{tabular}{|c|c|c|c|c|c|c|}
\hline Sprayers & $\varphi(\mathrm{deg})$ & $h(\mathrm{~mm})$ & $b(\mathrm{~mm})$ & $a(\mathrm{~mm})$ & $F\left(\mathrm{~mm}^{2}\right)$ & $\xi$ \\
\hline 1 & 60 & 9 & 8.38 & 19.19 & 110.50 & 0.35 \\
\hline 2 & 60 & 11.5 & 10.69 & 20.135 & 150.28 & 0.475 \\
\hline 3 & 90 & 4 & 5.045 & 13.31 & 45.81 & 0.10 \\
\hline 4 & 90 & 6.9 & 8.75 & 17.12 & 104.04 & 0.225 \\
\hline 5 & 90 & 9 & 11.67 & 19.03 & 157.10 & 0.35 \\
\hline 6 & 90 & 11.5 & 14.28 & 20.10 & 206.27 & 0.475 \\
\hline 7 & 120 & 9 & 14.72 & 19.105 & 209.06 & 0.35 \\
\hline
\end{tabular}

As a first step, the experiments have been made with a single sprayer for a fixed $A P$ and $H$. Then, two identical sprayers were used for the same parameters $A P, H$ with the addition of the parameter $L$ (nozzles spacing) to identify the dispersion of water in the intersection jets area (Fig. 4). The sprayers used in each experiment have identical geometric characteristics (twin jets) and are close to those actually used in industrial cooling systems (metallurgy).

The experimental data was classified in two groups; $1^{\text {st }}$ group represents the behavior of the fluid dispersion $(g)$ according to the $\mathrm{x}$ axis $(\mathrm{mm})$ only (one spatial dimension), $2^{\text {nd }}$ group represents the behavior of the fluid dispersion according to the $\mathrm{x}$ and $\mathrm{y}$ axis (two spatial dimensions).

\section{Equations governing the dispersion of water under- neath sprayers}

The dispersion of water droplets over a horizontal plate (Fig. 4) for single sprayer (TAB.1) was studied experimentally by Tebbal and Mzad, the authors established a correlation which can be described by the following relationship,

$$
g\left(x_{i}, y_{i}\right)=g_{\max }(0,0) \cdot \exp \left[-0.5\left(\frac{x_{i}^{2}}{x_{\max }^{2} \cdot \sigma_{x}^{2}}+\frac{y_{i}^{2}}{y_{\max }^{2} \cdot \sigma_{y}^{2}}\right)\right]
$$

$g\left(x_{\mathrm{i}}, y_{\mathrm{i}}\right)$ represents the fluid dispersion on the horizontal plate, while $g_{\max }(0,0)$ is the maximum fluid dispersion at the center of sprays, $x_{\mathrm{i}}$ and $y_{\mathrm{i}}$ are the spatial coordinates on the plate. $x_{\max }$ is a half of $D_{\text {large }}$ (radius) shown in (Fig. 4), $y_{\max }$ is the radius of the impacting water area by one sprayer on y'oy plan, $\sigma_{x}$ and $\sigma_{y}$ are the variances.

Where:

$$
g_{\max }(0,0)=2.1039 \cdot K_{1}^{1.206} \cdot \xi^{-0.702}
$$

\section{With:}

$$
K_{1}=F(2 \Delta P \cdot \rho)^{0.5} \cdot H^{-2} .
$$

And:

$$
y_{\max }=21.27 \cdot K_{2}^{0.062} \text {. }
$$

$K_{2}$ and $x_{\text {max }}$ are given by the relations (4) and (3) respectively as above. Where; the geometrical parameter $\xi$ was represented by the following relation;

$$
\xi=\frac{h-\delta}{2 R}
$$

With:

$$
\delta=\frac{-a}{2 \operatorname{tg}\left(\frac{\phi}{2}\right)}+h
$$

And:

$$
R=\frac{h^{2}+\delta^{2}-2 h \cdot \delta}{2(h-\delta)}+\frac{b^{2}}{8(h-\delta)}
$$

The variances $\sigma_{x}$ and $\sigma y$ present in the correlation (5) are given empirically and their values are 0.4304 and 0.4121 respectivelly Mzad and Elguerri carried out study based on the results of Tebbal and Mzad . The authors simulated twin-overlapping sprays underneath hydrodynamic atomizers and used the principle of simple addition in the intersection jets area on a horizontal plate. The summation law of both jets in this area was also used by other authors Hall and Mudawar; Hattel and Pryds and the dispersion in the intersection area of jets was described as following:

$$
g\left(x_{i}, y_{i}\right)=g_{\max }(0,0)\left[\exp \left[-0.5\left(\frac{\left(x-x_{1}\right)^{2}}{x_{\max }^{2} \cdot \sigma_{x}^{2}}+\frac{\left(y-y_{1}\right)^{2}}{y_{\max }^{2} \cdot \sigma_{y}^{2}}\right)\right]+\exp \left[-0.5\left(\frac{\left(x-x_{2}\right)^{2}}{x_{\max }^{2} \cdot \sigma_{x}^{2}}+\frac{\left(y-y_{2}\right)^{2}}{y_{\text {max }}^{2} \cdot \sigma_{y}^{2}}\right)\right]\right]
$$

where $\left(x_{1}, y_{1}\right)$ and $\left(x_{2}, y_{2}\right)$ are the center coordinates.

Some experimental studies were realized to quantify the dispersion of water in the overlapping area of the jets, while simulation study cannot accurately describe the phenomenon of dispersion due to the large number of hydrodynamics and geometrics parameters influencing the dispersion of liquid. The purpose of this study is to provide a base of experimental data and prove that the dispersion in 
this area is not subject to the addition law for the nozzles selected above (Table 1).

\section{Experimental results and discussion}

The $1^{\text {st }}$ group represents the behaviour of the fluid dispersion $(g)$ in the intersection area of jets on the plan $x$ 'ox only, the results were divided on three subgroups, each one includes its own geometrics and hydrodynamics parameters as describe below:

- $\quad 1^{\text {st }}$ subgroup includes the sprayers 2 and 5 (TAB.1), $H$ is of $455 \mathrm{~mm}, D_{\text {overlap }}$ is of $180 \mathrm{~mm}$ and $A P$ increases from 0.05 to $0.3 \mathrm{MPa}$ (Fig. 5).

- $\quad 2^{\text {nd }}$ subgroup includes the sprayers 1 and 4 (TAB.1), $H$ is of $385 \mathrm{~mm}, D_{\text {overlap }}$ is of $180 \mathrm{~mm}$ and $A P$ increases from 0.05 to $0.3 \mathrm{MPa}$ (Fig. 6).

- $\quad 3^{\text {rd }}$ subgroup includes the sprayers 2 and 6 (Table 1$), H$ is of $450 \mathrm{~mm}, D_{\text {overlap }}$ is of $210 \mathrm{~mm}$ and

- $\quad A P$ increases from 0.05 to $0.3 \mathrm{MPa}$ (Fig.7)

The experimental results of the fluid dispersion in the overlapping area of jets were represented in the Figs. 5; 6 and 7 for different hydrodynamic and geometric parameters as follow; pressure varies from 0.05 to $0.3 \mathrm{MPa}$, heights $H$ for the values 385, 450 and $455 \mathrm{~mm}$, overlapping diameter for 180 and $210 \mathrm{~mm}$ and different sprayers selected 1, 2, 4, 5 and 6 (Table 1).

The change of the the hydrodynamic and geometric parameters allows to have different flow rates which makes the analysis and the quantification of the fluid dispersion in the overlapping area of jets most credible.

Curves 1 and 2 are obtained for both sprayers (twin sprayer), curve 3 represents the dispersion obtained experimentally during the overlap of the two sprayers, curve 4 is the result of the simple addition of the two curves 1 and 2 (Figs. 5-7).

The number $K$ represents the relationship between the average experimental dispersion $\bar{g}_{\text {exp }}$ and the average dispersion obtained by the simple addition of both jets $\bar{g}_{\text {the }}$ in the overlapping area of jets. Where:

$$
K=\bar{g}_{\exp } / \bar{g}_{\text {the }} .
$$

With:

$$
\bar{g}_{\text {exp }}=\frac{\sum_{i=1}^{n} g_{\text {exp }}\left(x_{i}\right)}{n} .
$$

And:

$$
\bar{g}_{\text {the }}=\frac{\sum_{i=1}^{n} g_{\text {the }}\left(x_{i}\right)}{n} \text {. }
$$

$g_{\text {exp }}\left(x_{\mathrm{i}}\right)$ and $g_{\text {the }}\left(x_{\mathrm{i}}\right)$ are respectively the fluid dispersion obtained by experiments and by law of summation of both jets at all points on the x'ox plan in the overlapping area, illustrated by the curves 3 and 4 respectively (Figs. 5-7).

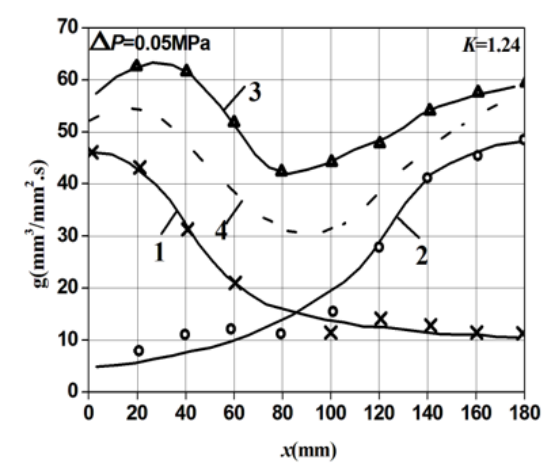

a

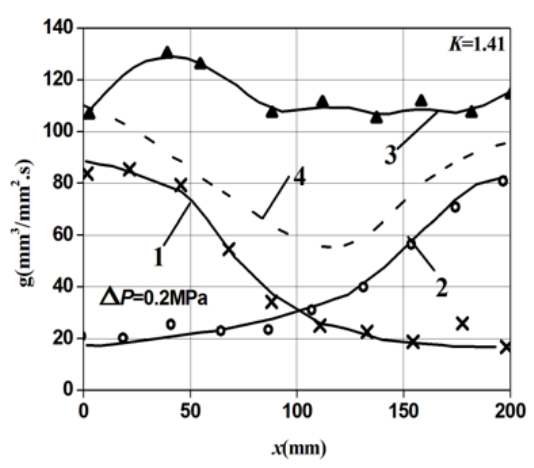

d

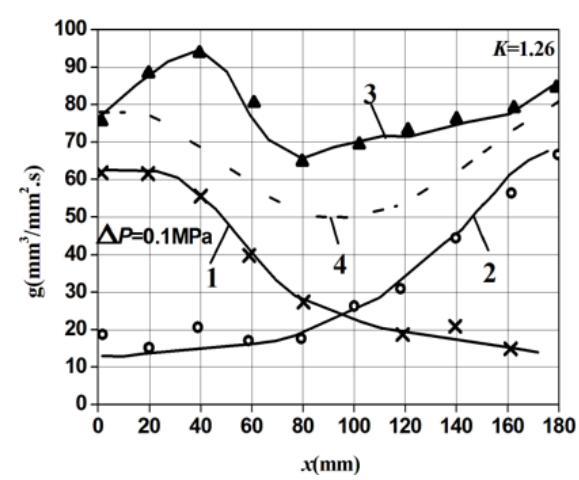

b

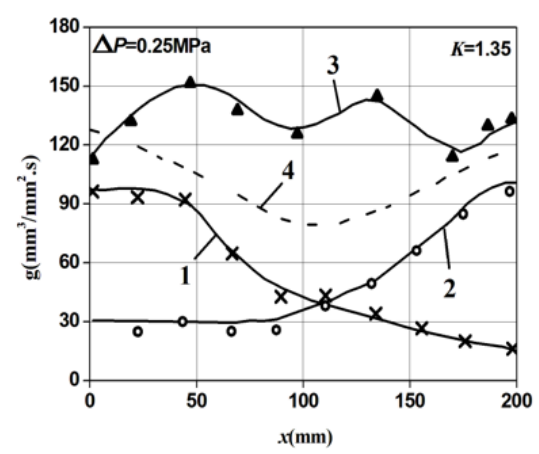

$\mathrm{e}$

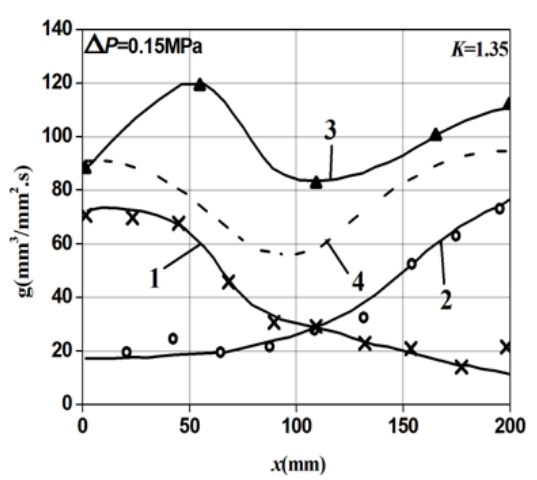

$\mathrm{C}$

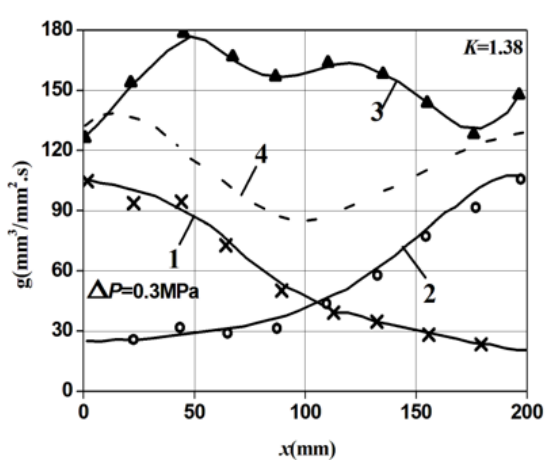

$\mathrm{f}$

Fig. 5 Behavior of the fluid dispersion on the x'ox plan for $D_{\text {overlap }}=180 \mathrm{~mm}, H=455 \mathrm{~mm}$, experimental data, group $01.1^{\text {st }}$ subgroup. Curve 1 and 2: dispersions for both sprayers ; curve 3: experimental dispersion ; curve 4: addition of curves

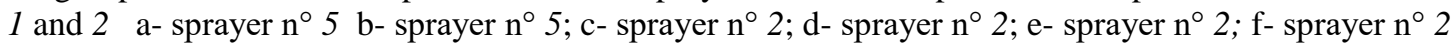




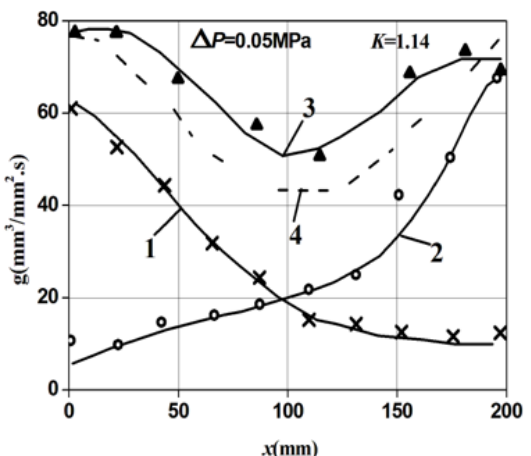

a

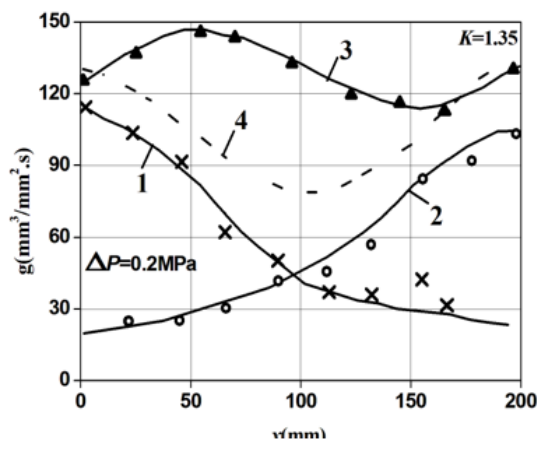

$\mathrm{d}$

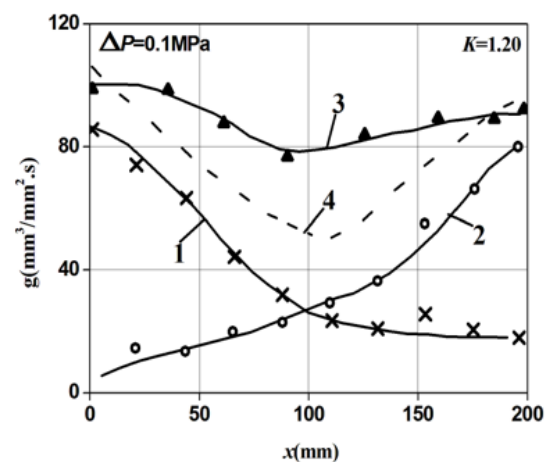

b

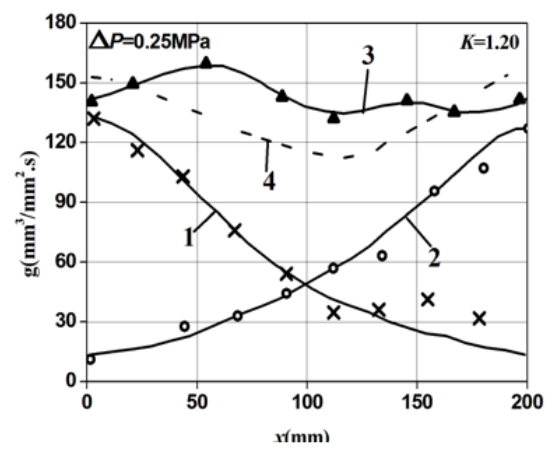

e

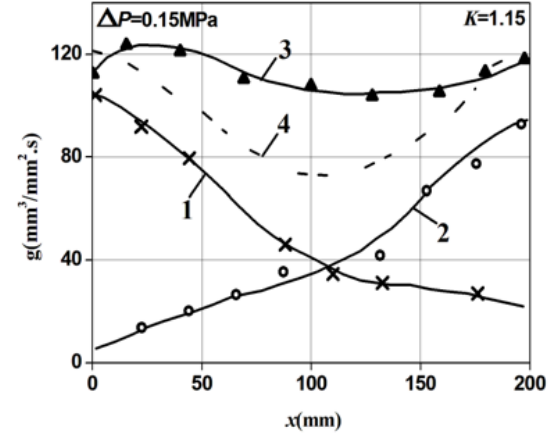

c

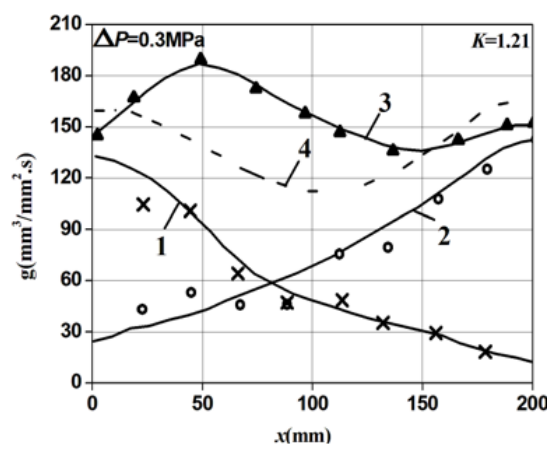

f

Fig. 6 Behavior of the fluid dispersion on the x'ox plan for $D_{\text {overlap }}=180 \mathrm{~mm}, H=385 \mathrm{~mm}$, experimental data, group $01,2^{\text {nd }}$ subgroup. Curve 1 and 2 : dispersions for both sprayers ; curve 3 : experimental dispersion ; curve 4 : addition of curves 1 and 2 a- $\operatorname{sprayer}^{\circ} 4$ b- $\operatorname{sprayer} \mathrm{n}^{\circ} 4$; $\mathrm{c}$ - $\operatorname{sprayer} \mathrm{n}^{\circ} 1 ; \mathrm{d}$ - $\operatorname{sprayer} \mathrm{n}^{\circ} 1$; e- $\operatorname{sprayer} \mathrm{n}^{\circ} 1 ; \mathrm{f}$ - $\operatorname{sprayer}^{\circ} 1$
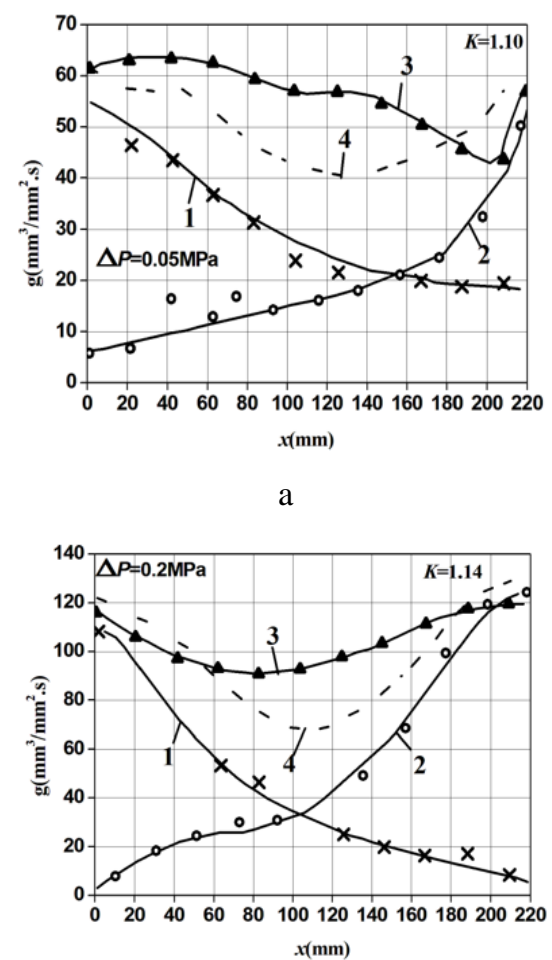

d

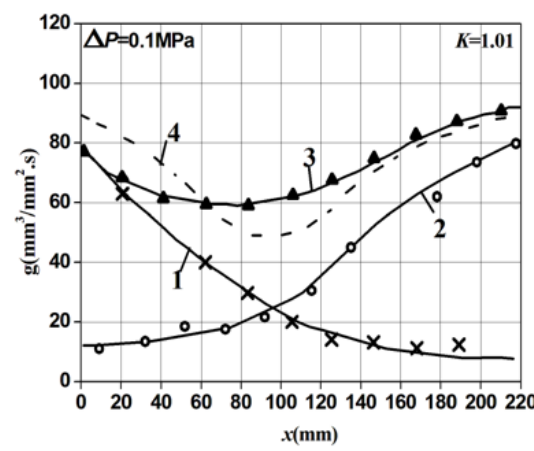

b

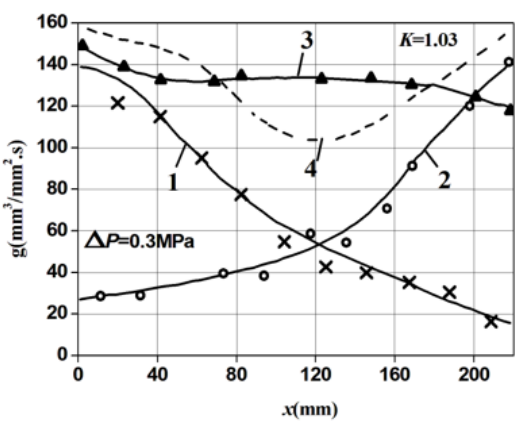

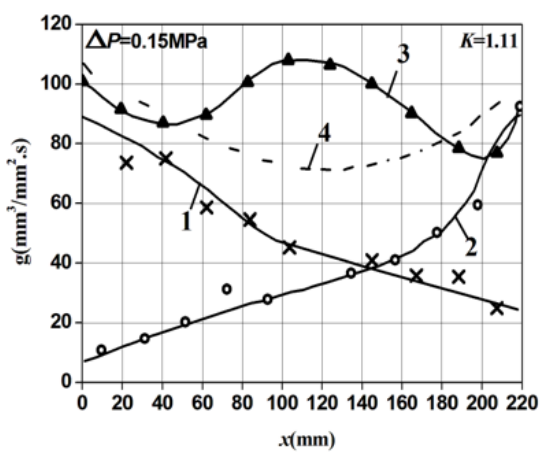

C

e

Fig. 7 Behavior of the fluid dispersion on the x'ox plan for $D_{\text {overlap }}=210 \mathrm{~mm}, H=450 \mathrm{~mm}$, experimental data, group $01.3^{\text {rd }}$ subgroup. Curve 1 and 2: dispersions for both sprayers ; curve 3: experimental dispersion ; curve 4: addition of curves 1 and 2 a- sprayer $n^{\circ} 6$ b- sprayer $^{\circ} 6$; c- sprayer $n^{\circ} 2 ; d-\operatorname{sprayer}^{\circ} 2$; e- $\operatorname{sprayer}^{\circ} 2$ 
The values of the dimensionless number $K$ for each test are illustrated on the graphical representations (Figs. 57) and collected in Table 2 as following.

Table 2

The values of the number $K$ on the x'ox plan

\begin{tabular}{|c|c|c|c|}
\hline \multirow{3}{*}{$\begin{array}{c}A P \\
(\mathrm{MPa})\end{array}$} & \multicolumn{3}{|c|}{$y=0 \mathrm{~mm}$} \\
\hline & $\begin{array}{c}D_{\text {overlap }}=180 \\
H=455 \\
(\mathrm{~mm})\end{array}$ & $\begin{array}{c}D_{\text {overlap }}=180 \\
H=385 \\
(\mathrm{~mm})\end{array}$ & $\begin{array}{c}D_{\text {overlap }}=210 \\
H=450 \\
(\mathrm{~mm})\end{array}$ \\
\hline & $K$ & $K$ & $K$ \\
\hline 0.05 & 1.24 & 1.14 & 1.10 \\
\hline 0.1 & 1.26 & 1.20 & 1.01 \\
\hline 0.15 & 1.35 & 1.15 & 1.11 \\
\hline 0.2 & 1.41 & 1.35 & 1.14 \\
\hline 0.25 & 1.35 & 1.20 & 1.12 \\
\hline 0.3 & 1.38 & 1.21 & 1.03 \\
\hline
\end{tabular}

The experimental dispersion illustrates by the curve 3 is the uppermost curve in the entirety of the graphical representations (Figs.5-7), that allows to deduce that the dispersion of the fluid obtained experimentally in the intersection jets area is more important and denser than that obtained by the summation law of both jets (curve 4 ).

The Table 2 gathers all the values of the number $K$, for all conditions. The values of the number $K$ are superior to the unit $(K>1)$ (Table 2) which proves that the dispersion of water droplets obtained experimentally (curve 3) is greater (more dense) than that obtained by summation law of both jets (curve 4) and the summation law was not checked for this type of sprayers (Table 1 ) for a fixed $H, L$ and different values of $A P$, the dispersion of water in the overlapping area of jets stays always more important than that obtained by summation law with a slow change when the pressure increases. The increase of the pressures from 0.05 to $0.3 \mathrm{MPa}$ has a slight influence on the values of $K$. This minimum change on the values of the number $K$ can be seen in each subgroup (Figs. 5-7) and in each column of the Table 2.

The nozzles spacing $L$ has a significant impact on the values of the number $K$; the three columns (Tab.2) show that the decrease of $D_{\text {overlap }}$ from 210 to $180 \mathrm{~mm}$ with a fixed pressure increases the value of the number $K$ (Table 2 ). We can also see that the value of $K$ is of 1.11 for $H=450 \mathrm{~mm}$, $D_{\text {overlap }}=210 \mathrm{~mm}$ and $A P=0.05 \mathrm{MPa}$, varies to 1.24 , for $H=455 \mathrm{~mm}, D_{\text {overlap }}=180 \mathrm{~mm}$ and $A P=0.05 \mathrm{MPa}$, which explains that the values may increase by square and this is the case for all other values of the two columns, three and one (Table 2).

The influence of the hydrodynamic parameter $H$ (distance between sprayer and plane surface) on the values of the number $K$ is seen in the two first columns (Table 2) when $L$ (nozzles spacing) is fixed at $180 \mathrm{~mm}$. The increase of $H$ improves the dispersion in the intersection area of jets. The explanation of the excess flow rate in the intersection area of jets remains to the merging of the two sprays. The decrease of $L$ brings closer the centers of the two sprayers where the dispersions are maximal $g_{\max }(0,0)$. The overlapping of the two jets causes a narrowing of the two large diameters of jets $\left(D_{\text {Large }}\right.$. This shrinkage is due to the high dispersion in the opposite direction to the decrease of $L$ of both jets. This quantity of water is added in the intersection area of jets, which makes the dispersion in this area greater than that obtained by the summation of the both jets.

The pressure has an influence on the sprays separately; the increase or decrease of the two jets dispersions depends on the intensity of the pressure. The effect of the pressure does not appear on the values of $K$ seeing that the relation between the two dispersions $g_{\exp }\left(x_{\mathrm{i}}\right)$ and $g_{\text {the }}\left(x_{\mathrm{i}}\right)$ remains almost constant.

The influence of $H$ on the dispersion in the intersection area of jets is evident; the increase of $H$ increases the liquid surface area of both jets, which increases the intersection area of jets.

The volumetric distribution of the water for each sprayer (curves $l$ and curve 2) for all figures (Figs. 5-7) had a maximum value near the center of the spray (edges of graphics) and decreased exponentially away from the center.

The opening angle of the sprayer and the pressure are the two parameters that affect the impact zone it is also a fact that with an increase in the nozzle opening angle both the width and the thickness of spraying develop. As a result, the impact area increases, therefore reducing the impact.

The experimental dispersion illustrates by the curve 3 takes high values for lower pressures at the edges of graphical representations (centers of the two sprays (all figures)) and it decreases and reaches minimum rates for high pressure values.

It appears on (Fig. 5) that if the pressure is high, the experimental dispersion (curve 3) has an undulating form, which generates a heterogeneous distribution on the wetted surface, which proves to be a disadvantage during intensive cooling of highly heated surfaces. On the other hand, if the pressure is low the dispersion is relatively homogeneous on the surface and the profile presents less corrugated aspect.

By reducing the height $H$ from $455 \mathrm{~mm}$ to $385 \mathrm{~mm}$ (Figs.5-6), the fluid distribution becomes more homogeneous (less undulations), and significant improvements could be made in both product quality and cost savings by reducing the spray height.

The second group of experiments was carried out in the xoy plan (2D), the aim is to extend our investigation and analyze the influence of the hydrodynamic and geometcric parameter in the overlapping area of the water dispersion with a change in the spatial condtions.

As the first group, the $2^{\text {nd }}$ group represents the behaviour of the fluid dispersion $(g)$ but on the plan xoy, the results were divided on two subgroups, each one includes its own geometrics and hydrodynamics parameters as describe below:

$1^{\text {st }}$ subgroup includes the sprayers $3 ; 5$ and 7 (Table 1$), H$ is of $450 \mathrm{~mm}, D_{\text {overlap }}$ is of $220 \mathrm{~mm}$ and $A P$ is of $0.1 \mathrm{MPa}$ (Fig. 8).

- $\quad 2^{\text {nd }}$ subgroup includes the sprayers $3 ; 5$ and 7 (Table 1$), H$ is of $350 \mathrm{~mm}, D_{\text {overlap }}$ is of $220 \mathrm{~mm}$ and $A P$ is of $0.1 \mathrm{MPa}$ (Fig. 9).

The curves shown in the second group (Figs. 8, 9) have the same definitions as the curves of the first group (Figs. 5, 6, 7). The experiments were carried out for a fixed pressure and $D_{\text {overlap }}$ with changing sprayers and values of $H$ for each subgroup. 


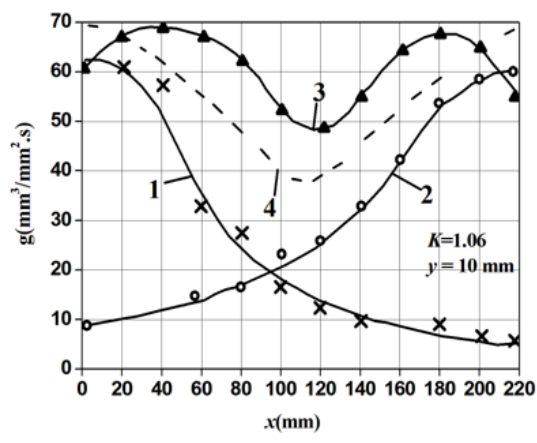

a

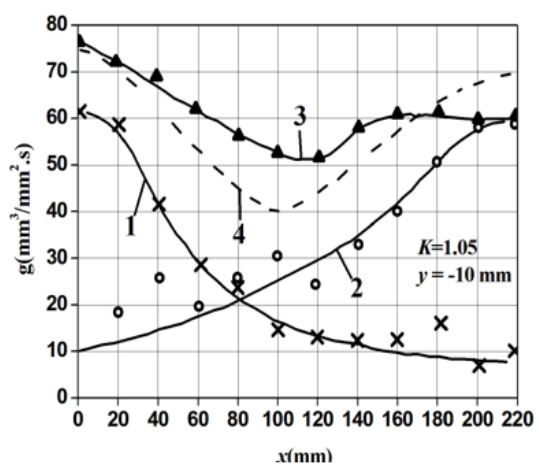

$\mathrm{c}$

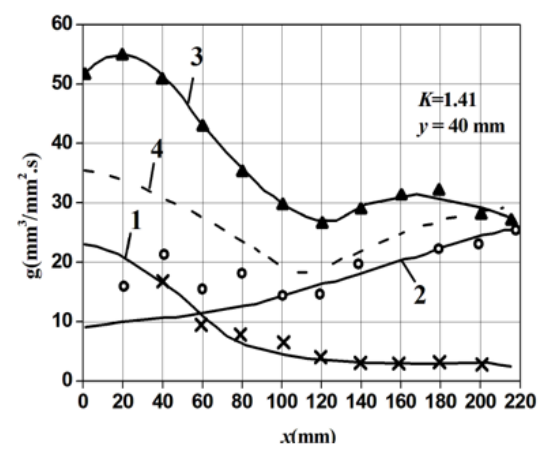

$\mathrm{b}$

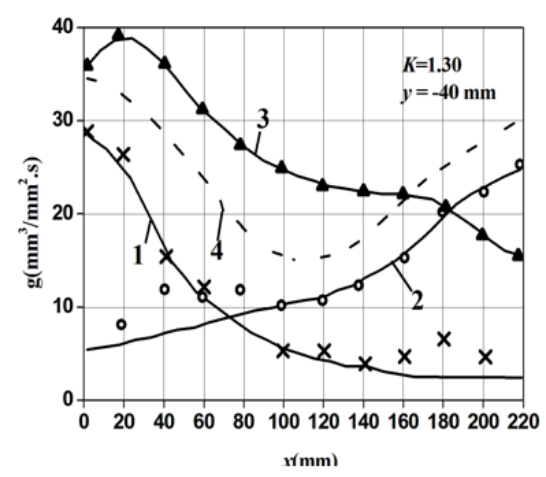

d

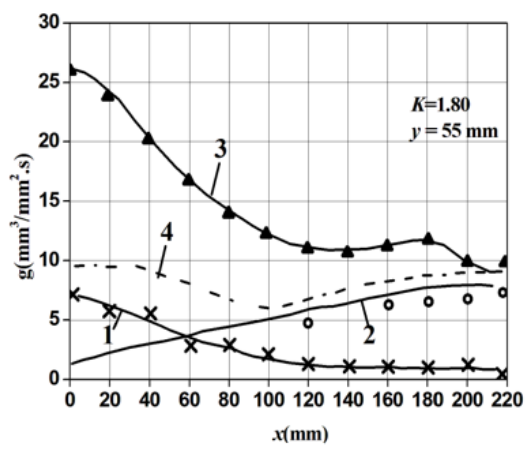

$\mathrm{c}$

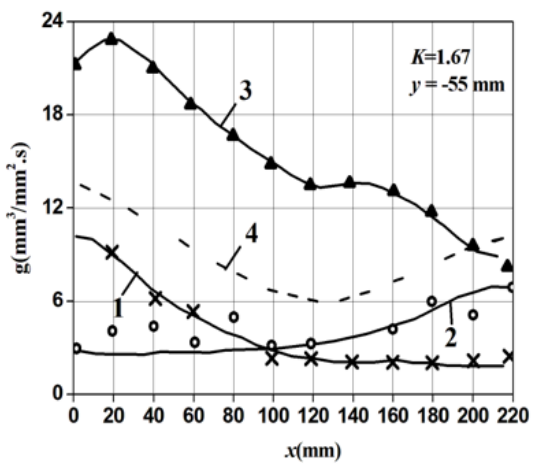

e

Fig. 8 Behavior of the fluid dispersion on the xoy plan for $D_{\text {overlap }}=220 \mathrm{~mm}, H=450 \mathrm{~mm}$, experimental data, group $02.1^{\text {st }}$ subgroup curve 1 and 2 : dispersions for both sprayers ; curve 3 : experimental dispersion; curve $4:$ addition of curves 1 and 2 a- sprayer $n^{\circ} 5$ b- sprayer $n^{\circ} 7$; c- sprayer $n^{\circ} 3$; d- sprayer $n^{\circ} 5$; e- sprayer $n^{\circ} 7 ; f$ - sprayer $n^{\circ} 3$

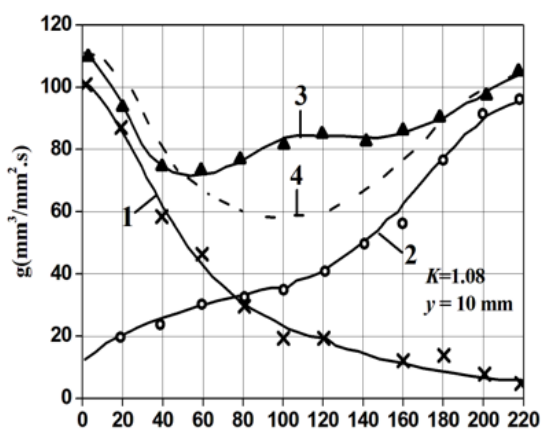

$x(\mathrm{~mm})$

a

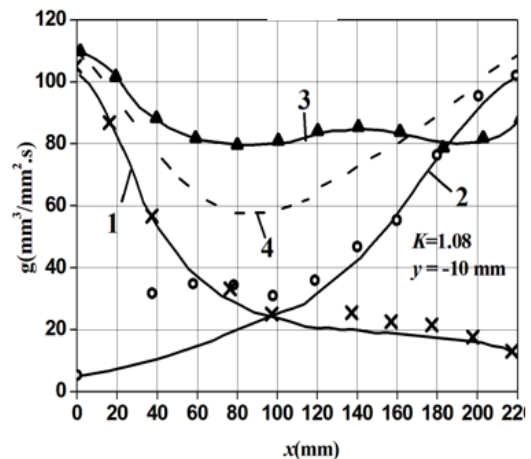

c

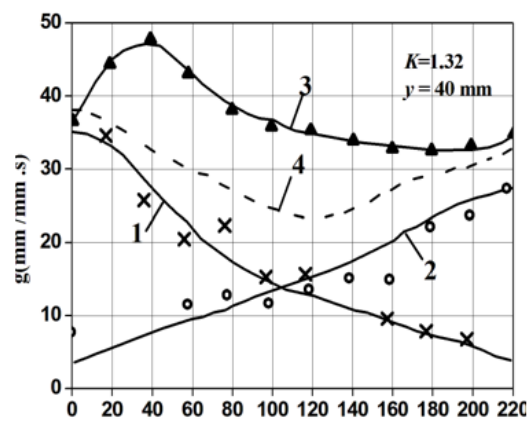

wmm)

b

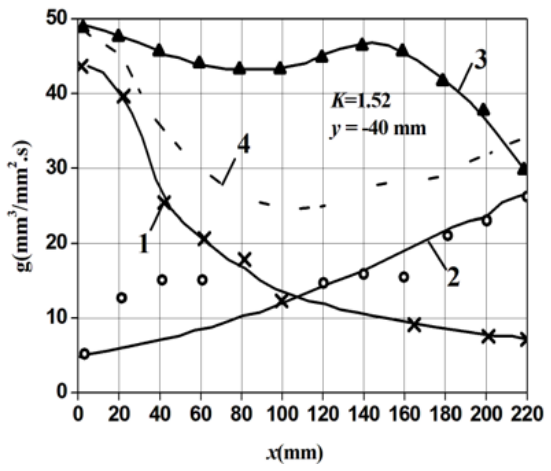

d

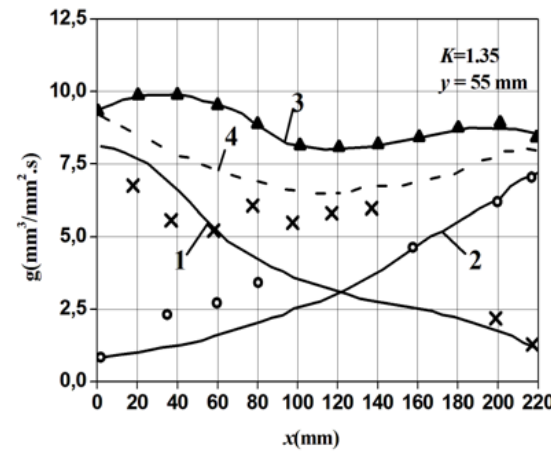

c

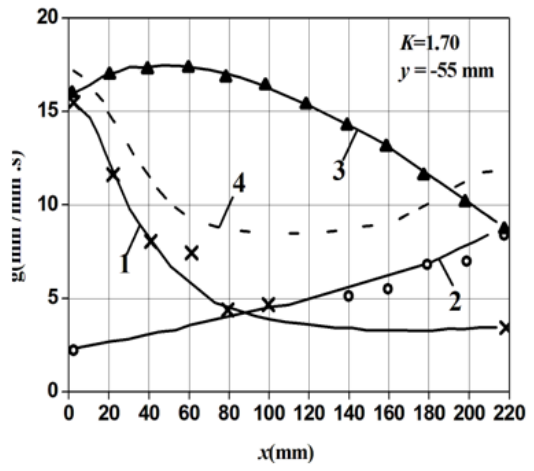

e

Fig. 9 Behavior of the fluid dispersion on the xoy plan for $D_{\text {overlap }}=220 \mathrm{~mm}, H=350 \mathrm{~mm}$, experimental data, group $02.2^{\text {nd }}$ subgroup. Curve 1 and 2: dispersions for both sprayers ; curve 3: experimental dispersion ; curve 4: addition of curves 1 and 2 a- sprayer $n^{\circ} 5$ b- sprayer $n^{\circ} 7$; c- sprayer $n^{\circ} 3 ; d$ - sprayer $n^{\circ} 5$; e- $\operatorname{sprayer} n^{\circ} 7 ; f-\operatorname{sprayer}^{\circ} 3$ 
The dimensionless number $K$ was calculated by the relation (13) where:

$$
\bar{g}_{\text {exp }}=\frac{\sum_{i=1}^{n} g_{\text {exp }}\left(x_{i}, y_{i}\right)}{n} .
$$

And:

$$
\bar{g}_{\text {the }}=\frac{\sum_{i=1}^{n} g_{\text {the }}\left(x_{i}, y_{i}\right)}{n} \text {, }
$$

$g_{\exp }\left(x_{i}, y_{i}\right)$ and $g_{\text {the }}\left(x_{i}, y_{i}\right)$ are respectively the fluid dispersion obtained by experiments and by law of summation of both jets at all points on the xoy plan in the overlapping area, illustrated by the curves 3 and 4 respectively (Figs. 8-9).

The values of the number $K$ for the group 2 (Figs. 8-9) were gathered on table (Table 3) as following;

Table 3

The values of the number $K$ on the xoy plan

\begin{tabular}{|c|c|c|}
\hline \multirow{2}{*}{$y(\mathrm{~mm})$} & \multicolumn{2}{|c|}{$A P=0.1 \mathrm{MPa}$} \\
\cline { 2 - 3 } & $\begin{array}{c}D_{\text {overlap }}=220 \mathrm{~mm} \\
H=450 \mathrm{~mm}\end{array}$ & $\begin{array}{c}D_{\text {overlap }}=220 \mathrm{~mm} \\
H=350 \mathrm{~mm}\end{array}$ \\
\cline { 2 - 3 } & $K$ & $K$ \\
\hline-55 & 1.67 & 1.70 \\
\hline-40 & 1.30 & 1.52 \\
\hline-10 & 1.05 & 1.08 \\
\hline 10 & 1.06 & 1.08 \\
\hline 40 & 1.41 & 1.32 \\
\hline 55 & 1.80 & 1.35 \\
\hline
\end{tabular}

We can see the extent of the values $K$ when the y'oy plan was added (Table 3). The largest increase of the number $K$ is of 1.80 (Table 3), which explains and cofirms that the dispersion of water in the intersection area of jets become more important and the addition law was not checked previously.

Finally we can duduct that the hydrodynamic overlap between the centers of sprayers ( the merging of sprayers) is the physical explanation of the non-additive character in the overlapping area of jets.

\section{Conclusion}

The quantification of the fluid dispersion underneath sprayers is still a big challenge in scientific literature because of the large influential numbers of hydrodynamic and geometric parameters. The numerical simulation tool is a helpful development mode but without experimental trials, it cannot predict the behaviour of this physical phenomenon, which was proved during our investigative study.

The analysis of the results for all the experiments leads to conclude that the experimental dispersion of the fluid in the intersection area of jets is more important (denser) than that obtained theoretically (summation of both jets) whatever the hydrodynamic and geometric conditions of the sprayers, which confirms that the principle of the simple addition was not checked previously.

The distance between the canters of sprayers $(L)$ was the most influential hydrodynamic parameter, the dispersion in the intersection area of jets increases when the water beams of the two sprayers approaches ( $L$ decreases) and it loses extent when $L$ increases but in all cases it is still more dense than the dispersion obtained by the summation law of both jets.

The investigation of geometrics and hydrodynamics parameters impact in the intersection jets area of the water dispersion on a horizontal plate was carried out in order to improve the heat transfer. Obviously the difference between the two dispersions; experimental and theoretical (summation law) causes a difference in the study of heat transfer intensity, the mastery and the suitable choice of hydrodynamics parameters is paramount, where; experimental data supplied in this study will be an important database for future works.

\section{References}

1 Lefebvre, A.H. 1989. Properties of sprays, Particle \& Particle Systems Characterization, 6: 176-186. http://dx.doi.org/10.1002/ppsc.19890060129.

2 Liu, H. 1999. Science and Engineering of Droplets: Fundamentals and Applications, William Andrew.

3 Nasr, G.G.; Yule, A.J.; Bendig, L. 2013. Industrial sprays and atomization: design, analysis and applications: Springer Science \& Business Media. http://dx.doi.org/10.1007/978-1-4471-3816-7.

4 Fritsching, U. 2004. Spray simulation: modeling and numerical simulation of sprayforming metals: Cambridge University Press. https://books.google.dz/books?id=Ol-x4ICbJD4C. https://doi.org/10.1017/CBO9780511536649.

5 Tebbal, M.; Mzad, H. 2004.An hydrodynamic study of a water jet dispersion beneath liquid sprayers, Forschung im Ingenieurwesen, 126-132,

http://dx.doi.org/10.1007/s10010-003-0118-3.

6 Mzad, H.; Tebbal, M. 2009. Thermal diagnostics of highly heated surfaces using water-spray cooling, Heat and Mass Transfer, 45: 287-295.

http://dx.doi.org/10.1007/s00231-008-0431-3

7 Cheng, W.-L.; Liu, Q.-N.; Zhao, R.; Fan, H.-I. 2010. Experimental investigation of parameters effect on heat transfer of spray cooling, Heat and mass transfer, 46: 911-921. http://dx.doi.org/10.1007/s00231-010-0631-5.

8 Visaria, M.; Mudawar, I. 2009. Application of twophase spray cooling for thermal management of electronic devices, IEEE Transactions on Components and Packaging Technologies, 32: 784-793. https://doi.org/10.1109/TCAPT.2008.2010405.

9 Chen, R.-H.; Chow, L.C.; Navedo, J. E. 2004. Optimal spray characteristics in water spray cooling, International Journal of Heat and Mass Transfer, 47: 5095-5099.

http://dx.doi.org/10.1016/j.ijheatmasstransfer.2004.05.0 33.

10. Chen, R.-H.; Chow, L. C.; Navedo, J. E. 2002. Effects of spray characteristics on critical heat flux in subcooled water spray cooling, International Journal of Heat and Mass Transfer, 45: 4033-4043. 
http://dx.doi.org/10.1016/S0017-9310(02)00113-8.

11. Celata, G.P.; Cumo, M;. Mariani, A.; Zummo, G. 2006. Visualization of the impact of water drops on a hot surface: effect of drop velocity and surface inclination, Heat and mass transfer 42: 885-890. http://dx.doi.org/10.1007/s00231-006-0139-1.

12 Abbasi, B.; Kim, J.; Marshall, A. 2010. Dynamic pressure based prediction of spray cooling heat transfer coefficients, International Journal of Multiphase Flow 36: 491-502.

http://dx.doi.org/10.1016/j.ijmultiphaseflow.2010.01.00 7.

13 Moreno, G.; You, S.M.; Steinthorsson, E. 2007. Spray cooling performance of single and multi-jet spray nozzles using subcooled FC-72, in ASME/JSME 2007 Thermal Engineering Heat Transfer Summer Conference collocated with the ASME 2007 InterPACK Conference, 783-790. http://doi.org/10.1115/HT2007-32429.

14 Horsk,Ý. J.; Raudenský, M. 2005. Measurement of heat transfer characteristics of secondary cooling in continuous casting, Metal 2005, 1-8.

15 Hou, Y.; Tao, Y.; Huai, X.; Guo, Z. 2012. Numerical characterization of multi-nozzle spray cooling, Applied Thermal Engineering 39: 163-170. http://dx.doi.org/10.1016/j.applthermaleng.2012.01.030

16 J.-W. Jung, K.-J. Cha, and D.-J. Kim. 2000. Characteristics of the droplet behavior in the overlap region of twin spray and in single spray, Transactions of the Korean Society of Mechanical Engineers B, vol. 24, 1300-1308.

http://dx.doi.org/10.22634/KSME-B.2000.24.10.1300.

17 Jurng, J.; Park, C.W.; Park, C.B. 1999. Imaging measurement of the structure of a twin overlapping spray for ice-slurry making, Atomization and Sprays, 9. http://dx.doi.org/10.1615/AtomizSpr.v9.i4.20.

18 Zhou, Y.; Lee, S.; McDonell, V.; Samuelsen, S.; Kozarek, R.L.; Lavernia, E.J. 1997. Influence of operating variables on average droplet size during linear atomization, Atomization and Sprays, 7. http://dx.doi.org/10.1615/AtomizSpr.v7.i4.10.

19 Patrick, B.; Barber, B.; Brown, D. 2001. Practical aspects of the design, operation and performance of caster spray systems, Revue de Métallurgie, 98: 383390. https://doi.org/10.1051/metal:2001192.

20 Hall, D.D.; Mudawar, I. 1995. Experimental and numerical study of quenching complex-shaped metallic alloys with multiple, overlapping sprays, International journal of heat and mass transfer 38: 1201-1216. http://dx.doi.org/10.1016/0017-9310(94)00244-P.

21 Hattel, J.; Pryds, N. 2004. A unified spray forming model for the prediction of billet shape geometry, Acta materialia 52: 5275-5288 http://dx.doi.org/10.1016/j.actamat.2004.07.016.

22 Mzad, H.; Elguerri, M. 2012. Simulation of twin overlapping sprays underneath hydraulic atomizers: influence of spray hydrodynamic parameters, Atomization and Sprays 22.

http://dx.doi.org/10.1615/AtomizSpr.2012006076.

K. Choual, R. Benzeguir, M. Tebbal

\section{EXPERIMENTAL STUDY OF THE DISPERSION \\ BENEATH LIQUID SPRAYERS IN THE INTERSECTION AREA OF JETS ON A HORIZONTAL PLATE}

S u m m a r y

The present paper is an experimental investigation of the hydrodynamic behaviour of two identical sprayers on a horizontal fixed plate, especially on the dispersion of water in the intersection area of jets. The aim of this work is to optimize heat transfer intensity by studying the dispersion of water droplets; we demonstrate experimentally that the fluid dispersion in the intersection jet area is different than the dispersion obtained by summation of both jets for different hydraulic atomizers.

Different hydrodynamic parameters were used with different variations as following; the water pressure $(A P)$ from 0.05 to $0.3 \mathrm{MPa}$, the distance between sprayer and surface of plate $(H)$ from 385 to $450 \mathrm{~mm}$ and the nozzles spacing $(L)$ was calculated to maintain the overlapping diameter (Dovelap) of jets on plate between 180 and $220 \mathrm{~mm}$.

Keywords: sprayers, fluid dispersion, hydrodynamic, intersection area, quantification.

Received December 10, 2016 Accepted December 07, 2017 